Pedro Oguri

\title{
Aprendizado de Máquina para o Problema de Sentiment Classification
}

Dissertação apresentada como requisito parcial para obtenção do grau de Mestre pelo Programa de Pós-graduação em Mestrado em Informática do Departamento de Informática da PUC-Rio

Orientador : Prof. Ruy Luiz Milidiú Co-Orientador: Prof. Raúl Rentería 
Pedro Oguri

\title{
Aprendizado de Máquina para o Problema de Sentiment Classification
}

Dissertação apresentada como requisito parcial para obtenção do grau de Mestre pelo Programa de Pós-graduação em Mestrado em Informática do Departamento de Informática do Centro Técnico Científico da PUC-Rio. Aprovada pela Comissão Examinadora abaixo assinada.

\author{
Prof. Ruy Luiz Milidiú \\ Orientador \\ Departamento de Informática - PUC-Rio
}

Prof. Raúl Rentería

Co-Orientador

Departamento de Informática - PUC-Rio

Prof. Marcus Vinícius Soledade Poggi de Aragão

Departamento de Informática - PUC-Rio

Prof. Marco Antonio Casanova

Departamento de Informática - PUC-Rio

Prof. José Eugênio Leal Coordenador Setorial do Centro Técnico Científico - PUC-Rio 
Todos os direitos reservados. É proibida a reprodução total ou parcial do trabalho sem autorização da universidade, do autor e do orientador.

\section{Pedro Oguri}

Graduou-se Bacharel em Informática pela PUC-Rio em 2003 tendo cursado parte de sua graduação na Universidad Autonoma de Madrid. Trabalhou como Engenheiro de Software na Accenture e na Fast Search and Transfer. Tem interesse na pesquisa de algoritmos, especialmente nas áreas de aprendizado de máquina e otimização combinatória.

Oguri, Pedro

/ Pedro Oguri; orientador: Ruy Luiz Milidiú; coorientador: Raúl Rentería. - Rio de Janeiro : PUC-Rio, Departamento de Informática, 2006.

v., 54 f: il. ; $29,7 \mathrm{~cm}$

1. Dissertação (mestrado) - Pontifícia Universidade Católica do Rio de Janeiro, Departamento de Informática.

Inclui referências bibliográficas.

1. Informática - Tese. I. Milidiú, Ruy Luiz. II. Renteria, Raúl. III. Pontifícia Universidade Católica do Rio de Janeiro. Departamento de Informática. IV. Título. 


\section{Agradecimentos}

À minha esposa Vanessa e ao meu filho Breno, pelo apoio incondicional e compreensão.

Aos meus pais e à minha irmã que tanto me apoiaram e incentivaram.

Ao meu orientador Ruy Milidiú por ter me despertado tamanho fascínio pelo estudo de algoritmos através de seus ensinamentos.

Ao meu co-orientador e amigo Raul Rentería pelo apoio, incentivo e valiosas discussões.

Ao professor Marcus Poggi pelos ensinamentos, incentivo e entusiasmo contagiante.

Aos brasileiros que pagam impostos, por me sustentarem durante o primeiro ano do meu mestrado. 


\section{Resumo}

Oguri, Pedro; Milidiú, Ruy Luiz; Renteria, Raúl. Aprendizado de Máquina para o Problema de Sentiment Classification. Rio de Janeiro, 2006. 54p. Dissertação de Mestrado — Departamento de Informática, Pontifícia Universidade Católica do Rio de Janeiro.

Sentiment Analysis é um problema de categorização de texto no qual desejase identificar opiniões favoráveis e desfavoráveis com relação a um tópico. Um exemplo destes tópicos de interesse são organizações e seus produtos. Neste problema, documentos são classificados pelo sentimento, conotação, atitudes e opiniões ao invés de se restringir aos fatos descritos neste.

O principal desafio em Sentiment Classification é identificar como sentimentos são expressados em textos e se tais sentimentos indicam uma opinião positiva (favorável) ou negativa (desfavorável) com relação a um tópico. Devido ao crescente volume de dados disponível na Web, onde todos tendem a ser geradores de conteúdo e expressarem opiniões sobre os mais variados assuntos, técnicas de Aprendizado de Máquina vem se tornando cada vez mais atraentes.

Nesta dissertação investigamos métodos de Aprendizado de Máquina para Sentiment Analysis. Apresentamos alguns modelos de representação de documentos como saco de palavras e N-grama. Testamos os classificadores SVM (Máquina de Vetores Suporte) e Naive Bayes com diferentes modelos de representação textual e comparamos seus desempenhos.

\section{Palavras-chave}

Aprendizado de Máquina. Sentiment Analysis. Classificação de Texto. Classificadores Bayesianos. Support Vector Machines. 


\section{Abstract}

Oguri, Pedro; Milidiú, Ruy Luiz; Renteria, Raúl. Machine Learning for Sentiment Classification. Rio de Janeiro, 2006. 54p. MsC Thesis - Department of Informática, Pontifícia Universidade Católica do Rio de Janeiro.

Sentiment Analysis is a text categorization problem in which we want to identify favorable and unfavorable opinions towards a given topic. Examples of such topics are organizations and its products. In this problem, documents are classifed according to their sentiment, connotation, attitudes and opinions instead of being limited to the facts described in it.

The main challenge in Sentiment Classification is identifying how sentiments are expressed in texts and whether they indicate a positive (favorable) or negative (unfavorable) opinion towards a topic. Due to the growing volume of information available online in an environment where we all tend to be content generators and express opinions on a variety of subjects, Machine Learning techniques have become more and more attractive.

In this dissertation, we investigate Machine Learning methods applied to Sentiment Analysis. We present document representation models such as bag-of-words and N-grams. We compare the performance of the Naive Bayes and the Support Vector Machine classifiers for each proposed model.

\section{Keywords}

Machine Learning. Sentiment Analysis. Text Classification. Bayesian Classifiers. Support Vector Machines. 


\section{Sumário}

$\begin{array}{lll}1 & \text { Introdução } & 11\end{array}$

\begin{tabular}{lll}
\hline 1.1 & Objetivos da Dissertação & 11
\end{tabular}

1.2 Organização da Tese 12

$\begin{array}{llr}2 & \text { Sentiment Analysis } & 13\end{array}$

$\begin{array}{lll}2.1 & \text { Definição do Problema } & 13\end{array}$

\begin{tabular}{lll}
\hline 2.2 & Trabalhos Relacionados & 14
\end{tabular}

$\begin{array}{lll}3 & \text { Modelagem Textual } & 18\end{array}$

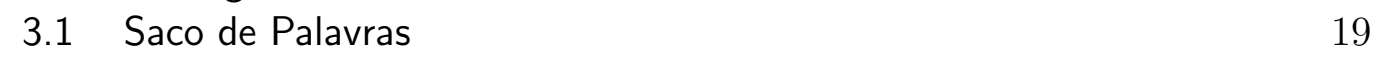

$\begin{array}{lll}3.2 & \text { N-gramas } & 19\end{array}$

3.3 Part of Speech Tagging 20

$\begin{array}{lll}3.4 & \text { Filtro de Subjetividade } & 20\end{array}$

3.5 Seleção de Features 21

3.5 .1 Informação Mútua Média 23

4 Classificador Naive Bayes $\quad 25$

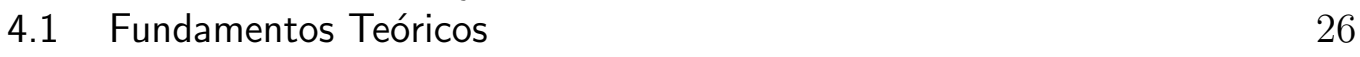

\begin{tabular}{ll}
4.1 .1 & Modelo Binário \\
\hline
\end{tabular}

\begin{tabular}{lll}
\hline 4.1 .2 & Modelo Multinomial & 27
\end{tabular}

$\begin{array}{lll}5 & \text { Classificador SVM } & 29\end{array}$

$\begin{array}{lll}5.1 & \text { Fundamentos Teóricos } & 29\end{array}$

5.1 .1 A dimensão VP e o limite do risco esperado 30

5.2 Máquinas de Vetores Suporte Lineares 30

$\begin{array}{lll}5.2 .1 & \text { Formulação Lagrangeana } & 32\end{array}$

5.3 Máquinas de Vetores Suporte Não-Lineares 33

6 Experimentos $\quad 35$

\begin{tabular}{lll}
\hline 6.1 & Corpus & 36
\end{tabular}

\begin{tabular}{lll}
\hline 6.2 & Metodologia de Teste & 36
\end{tabular}

\begin{tabular}{lll}
\hline 6.3 & Descrição dos Experimentos & 37
\end{tabular}

\begin{tabular}{|lll}
\hline 6.3 .1 & Corpus com Subjetividade Filtrada & 38
\end{tabular}

$\begin{array}{lll}6.4 & \text { Resultados } & 38\end{array}$

6.4.1 Discussão dos Resultados 40

\begin{tabular}{lll}
\hline 7 & Conclusão & 44
\end{tabular}

\begin{tabular}{lll}
\hline A & Resultados Detalhados & 46
\end{tabular}

B Fluxograma do Ambiente de Experimentação 50

\begin{tabular}{ll}
\hline Referências Bibliográficas & 52
\end{tabular} 


\section{Lista de figuras}

2.1 Processo de Classificação de Sentimento 14

5.1 Solução para um cenário de 2 dimensões linearmente separável. Os pontos pelos quais a linha pontilhada passa são chamados de vetores suporte

$\begin{array}{lll}6.1 & \text { Validação Cruzada com } 3 \text { folds } & 37\end{array}$

\begin{tabular}{lll}
\hline 6.2 & Desempenho do Classificador NB & 39
\end{tabular}

\begin{tabular}{lll}
\hline B.1 & Fluxograma da Solução & 51
\end{tabular} 


\section{Lista de tabelas}

2.1 Resultados reportados em Pang et al. (19) 15

3.115 features de IMM mais alto 24

6.1 Estatísticas do movie review data set 36

6.2 Estatísticas do corpus com subjetividade filtrada 38

6.3 Melhores precisões atingidas por cada classificador para cada modelo de representação de documentos 43

\begin{tabular}{lll}
\hline A.1 & Resultados para unigramas do corpus original & 46
\end{tabular}

$\begin{array}{lll}\text { A.2 } & \text { Resultados para bigramas do corpus original } & 47\end{array}$

A.3 $\quad$ Resultados para unigramas com classe gramatical (part of speech) 47

A.4 Resultados para bigramas com classe gramatical (part of speech) 48

A.5 Resultados para unigramas com subjetividade filtrada 48

A.6 Resultados para bigramas com subjetividade filtrada 49 
Science is what we understand well enough to explain to a computer. Art is everything else we do.

Donald Knuth, Prefácio do livro $A=B$. 